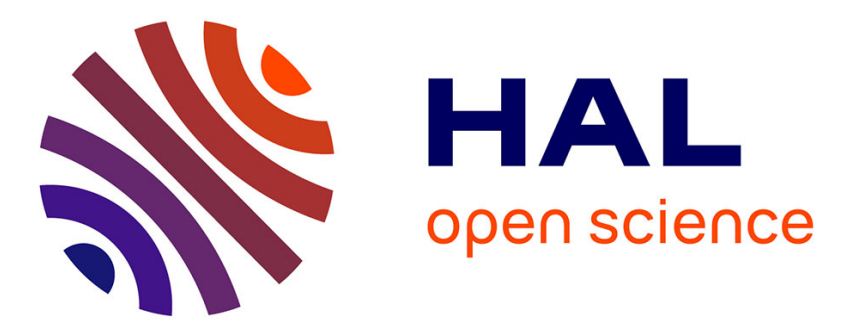

\title{
Planning acetabular fracture reduction using patient-specific multibody simulation of the hip
}

Hadrien Oliveri, Mehdi Boudissa, Jerome Tonetti, Matthieu Chabanas

\section{To cite this version:}

Hadrien Oliveri, Mehdi Boudissa, Jerome Tonetti, Matthieu Chabanas. Planning acetabular fracture reduction using patient-specific multibody simulation of the hip. SPIE Medical Imaging, Feb 2017, Orlando, United States. 10.1117/12.2250380 . hal-01490266

\section{HAL Id: hal-01490266 https://hal.science/hal-01490266}

Submitted on 15 Mar 2017

HAL is a multi-disciplinary open access archive for the deposit and dissemination of scientific research documents, whether they are published or not. The documents may come from teaching and research institutions in France or abroad, or from public or private research centers.
L'archive ouverte pluridisciplinaire HAL, est destinée au dépôt et à la diffusion de documents scientifiques de niveau recherche, publiés ou non, émanant des établissements d'enseignement et de recherche français ou étrangers, des laboratoires publics ou privés. 


\title{
Planning acetabular fracture reduction using patient-specific multibody simulation of the hip
}

\author{
Hadrien Oliveri ${ }^{\mathrm{a}}$, Mehdi Boudissa ${ }^{\mathrm{a}, \mathrm{b}}$, Jerome Tonetti ${ }^{\mathrm{a}, \mathrm{b}}$, Matthieu Chabanas*a \\ aTIMC-IMAG, Univ. Grenoble Alpes, CNRS, F-38000 Grenoble, France \\ ${ }^{b}$ Orthopedic and Traumatology Surgery, Grenoble Alpes Univ. Hospital, F-38000 Grenoble, France
}

\begin{abstract}
Acetabular fractures are a challenge in orthopedic surgery. Computer-aided solutions were proposed to segment bone fragments, simulate the fracture reduction or design the osteosynthesis fixation plates. This paper addresses the simulation part, which is usually carried out by freely moving bone fragments with six degrees of freedom to reproduce the pre-fracture state. Instead we propose a different paradigm, closer to actual surgeon's requirements: to simulate the surgical procedure itself rather than the desired result. A simple, patient-specific, biomechanical multibody model is proposed, integrating the main ligaments and muscles of the hip joint while accounting for contacts between bone fragments. Main surgical tools and actions can be simulated, such as clamps, Schanz screws or traction of the femur. Simulations are computed interactively, which enables clinicians to evaluate different strategies for an optimal surgical planning. Six retrospective cases were studied, with simple and complex fracture patterns. After interactively building the models from preoperative CT, gestures from the surgical reports were reproduced. Results of the simulations could then be compared with postoperative CT data. A qualitative study shows the model behavior is excellent and the simulated reductions fit the observed data. A more quantitative analysis is currently being completed. Two cases are particularly significant, for which the surgical reduction actually failed. Simulations show it was indeed not possible to reduce these fractures with the chosen approach. Had our simulator being used, a better planning may have avoided a second surgery to these patients.
\end{abstract}

Keywords: acetabular fracture, pelvic surgery, surgical simulation, virtual surgical planning, biomechanical modeling

\section{INTRODUCTION}

Acetabulum is the concavity of the pelvis that hosts the femoral head to form the hip joint. It is made up as the junction of three bones: the ilium, the ischium and the pubis. Many fracture patterns may occur [1], generally in a polytraumatic context. Surgical treatment is usually required to reduce the fracture then stabilize the pelvis via osteosynthesis. The quality of the reduction is the main prognostic factor for a good clinical outcome. A joint surface incongruence of less than $2 \mathrm{~mm}$ is generally considered acceptable, to avoid osteoarthritis leading to total hip replacement.

The first difficulty is to fully understand the fracture, even with high quality 3D CT reconstruction nowadays available (Figure 1, left). A planning must then be precisely set, defining which surgical approach to choose, how fragments will be repositioned and fixed, and in what order. Finally, the surgery itself is technically challenging. The acetabulum is located in a deep anatomical area surrounded by numerous vascular and nervous elements. Surgical approaches are narrow, with a very limited field of view, thus bone fragments and fracture lines are only partially visible. In addition, the articular surface on which rely the congruence success criteria is never directly accessible to the surgeon, neither during the reduction nor for its control. In a recent study, unsatisfactory results were found in $37 \%$ of cases [2].

Recent developments in imaging and computer technology have provided three main contributions for acetabular surgery: A) segmenting bone fragments from CT, B) simulating the fracture reduction and C) pre-forming the osteosynthesis fixation plates. Their goal is to provide surgeons with a simulator that can be used to evaluate different strategies to finally define an optimal surgical planning.

*Matthieu.Chabanas@univ-grenoble-alpes.fr 


\section{METHODOLOGY}

This paper addresses problem B), namely the crucial stage of surgery (and thus of virtual simulation) that consists in repositioning each bone in its anatomical position, i.e. the one before the fracture occurred.

\subsection{Paradigm}

Most authors propose to perform virtual reduction through geometrical repositioning of each bone fragment with 6 degrees of freedom $[3,4,5,6]$. However, manipulating 3D meshes in a $2 \mathrm{D}$ screen through mouse interaction or haptic device is very difficult. Moreover, fragments are moved freely with few or no anatomical consideration. The essential drawback of all these methods is that their goal is simply to produce a final geometric result instead of the clinical process to achieve it. Even if their target position is in fine correct, this does not yield to a real surgical planning. Our new paradigm is then to simulate the procedure itself, instead of the desired outcome. The aim is to define the best surgical approach and succession of gestures (compressions, distensions, tractions ...) to obtain a satisfying reduction, while accounting for the feasibility of the planning.

Pelvic surgery is by nature highly mechanical. Bone fragments are repositioned using clamps, hooks or screws, hence via the application of forces by the physician. Strong constraints are applied, due to the ligament and muscular system or contacts between structures during the reduction. To account for these elements, a biomechanical approach is proposed. First, a patient-specific model of the hip has to be built, integrating the bone fragments and a simplified representation of the anatomy. Then, the actions of virtual surgical instruments must be interactively simulated so that the surgeon can try and evaluate various strategies and finally define an optimal planning.

\subsection{Modeling the patient anatomy}

Many hip musculoskeletal models exist in the literature. Simplified representation using action-lines have proven to be useful for many applications [7], especially kinematic studies. More complex models, often relying on finite elements, are also used to compute constraints on bones or soft tissues $[8,9,10]$. However, few are patient-specific and none take fractures into account.

Using simulation in a surgical planning context imposes critical requirements. First, the model must be patient-specific and generated in very limited time. Second, the required elements may differ from one patient to the other depending on the fracture location and ligaments damages. Finally, simulations must be performed interactively. For all these reasons, we have chosen a very simple model using rigid bodies and action-lines springs. Despite the fact that anatomy is approximated in a very coarse way, this approach has proven to be relevant in our context. This model is implemented using the ArtiSynth framework [11].
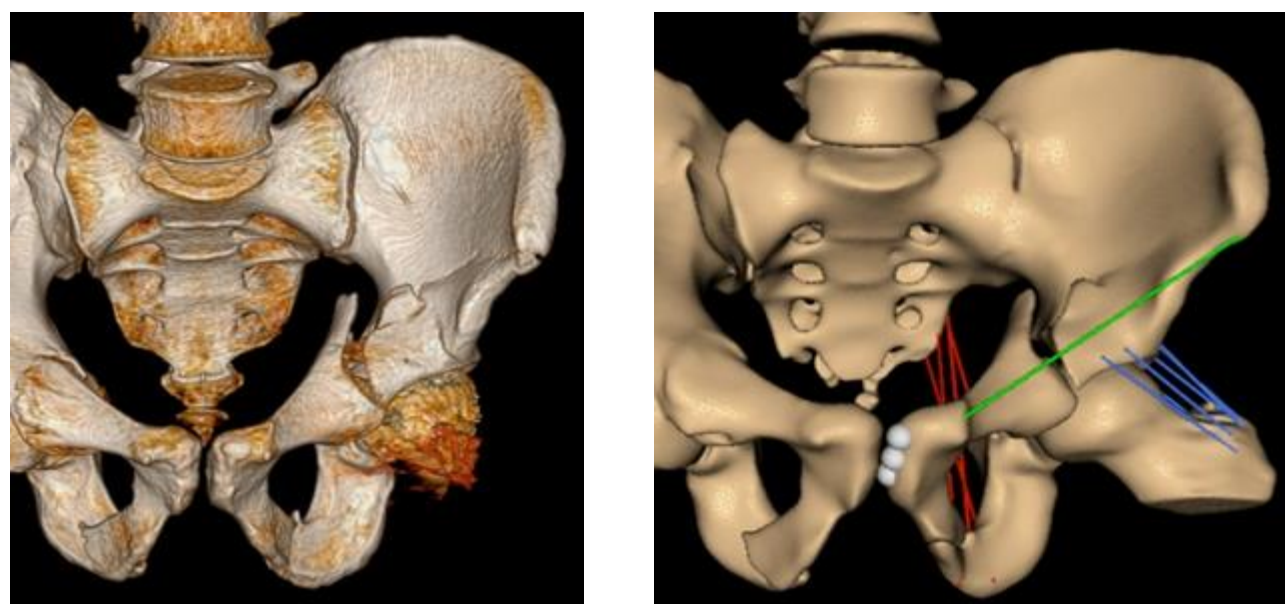

Figure 1. Clinical case \#6, a bi-columns fracture with comminuted fragments. Left: preoperative CT reconstruction. Right: multibody model of the patient including bone fragments, the pubic symphysis (in grey), the sacrotuberous ligament (posterior, in red), the ilioinguinal ligament (anterior, in green) and non-injured fibers of the hip capsule (in blue). 
The pelvic anatomical environment is extremely rich and complex. Hence, it cannot be entirely modeled with accuracy, especially in a patient-specific context. We have thus focused on elements that are the most relevant for our application. Each bony structure is represented by a separate rigid body, typically one for the femur, one for the main non-displaced bones and one for each displaced element and comminuted fragment. The main mechanical constraints in our context are due to ligaments. According to us, the most influential elements are the sacrotuberous ligament, the ilioinguinal ligament and the hip capsule. The pubic symphysis is also preponderant as it strongly resists to relative motion between the two pubic bones. Each ligament is modeled by a very limited number of point-to-point springs (Figure 1, right), resisting in extension but with a null response in compression. The aim is not to reproduce the anatomy with fidelity but only to consider the main direction of forces that are exerted to the bones. While it is obviously non-realistic, this basic model still gives a good approximation of the constraints exerted on the acetabulum. It may not be sufficient to model the hip kinematics, but it is suitable in our context. Parameters (bone density, ligaments stiffness, etc.) are taken from the literature. Acetabular surgery is performed under general anesthesia with injection of curare. Muscles are therefore relaxed and do not directly exert forces on the bone structures. So far, we have chosen not to represent muscles individually, alike the ligaments. Instead, the muscular environment is taken into account as a global strong damping applied to all bony structures. Finally, contacts are handled to ensure non-penetration between bone segments, with the femoral head or between fragments.

\subsection{Simulating surgical gestures}

During surgery, two classes of interactions could be realized to reduce the fracture. The first one apply compression or distraction between bone segments to bring them closer or away, using clamps or fixation screws. This is simulated using coaxial forces applied on two insertion points, one on each fragment, whose magnitude is interactively controlled. An example is shown Figure 2. The second class of actions regroups direct pulling or pushing of bone fragments, manually or using hooks or manipulating screws inserted into the bones. One extremity of a traction spring is fixed to a bony fragment, while the other is moved freely through mouse interaction (Figure 3).
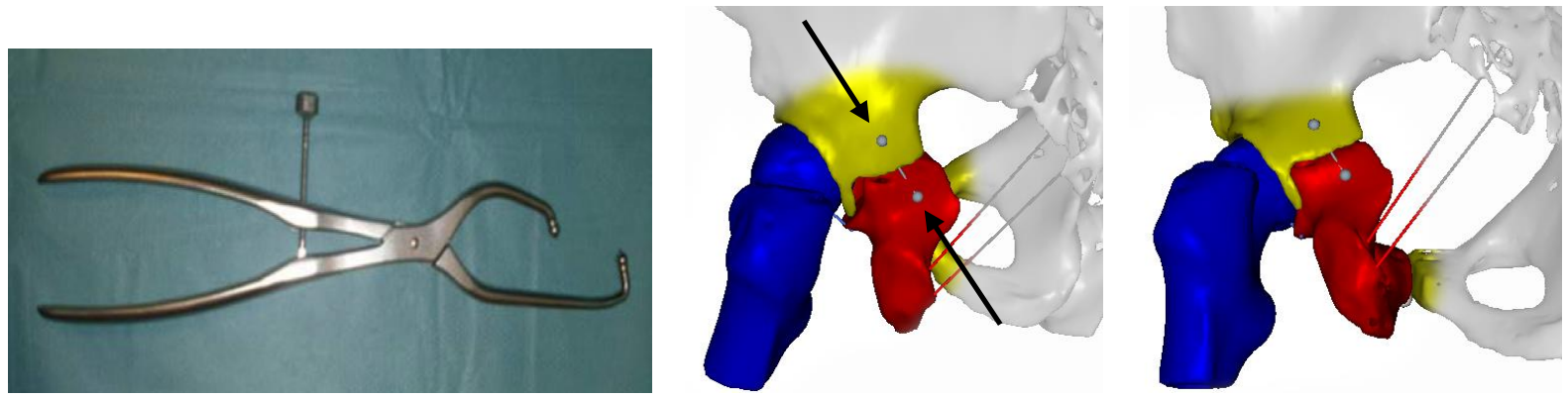

Figure 2. Matta clamp and its simulated effect on clinical case \#1, a simple transverse fracture. The ischium fragment is pulled toward the fixed ilium. The reduction was adequate, both in our simulation and after surgery.
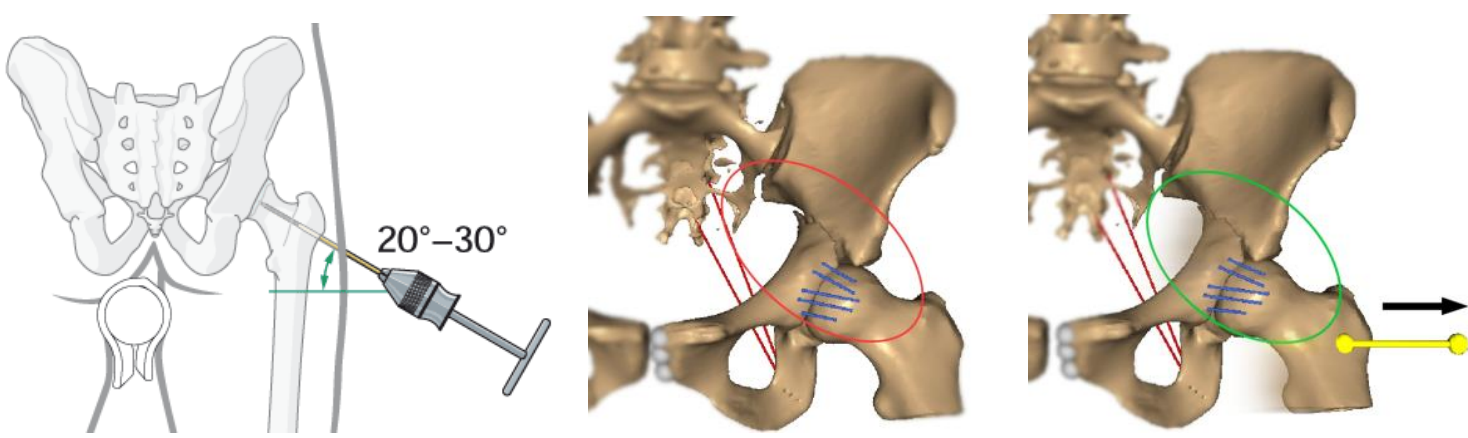

Figure 3. Schanz screw (image from the AO Fondation) and the simulation of femoral traction on a transverse fracture. Before traction, the head of femur is impacted towards the joint. After traction, the head of femur is in its correct position. Note that the capsule ligaments have partially brought the ischium towards the ilium, which is an observed behavior and the first step in the fracture reduction process. 


\section{RESULTS}

At this stage of the project, our goal is to evaluate the proposed model and the global behavior of the simulations. Ongoing works will later address the clinical relevance of our approach as an aid to define an optimal surgical planning.

\subsection{Retrospective validation}

Six retrospective cases with simple or complex fractures were studied. First, a model of the patient is built from preoperative CT images. Surgery is then simulated according to the surgical report, to reproduce the gestures that were actually realized. Finally, results of the simulation are compared to postoperative X-rays or CT data. Our aim is to qualitatively evaluate in what extent the simulation fits the actual data.

A simple reduction of transverse fracture, case \#1, is presented Figure 2. Case \#2 is especially interesting: this patient was first operated with a posterior Kocher-Langenbeck approach, with a single Jungbluth forceps. However, the femoral head was in fact impacted into the acetabular concavity, making the reduction impossible. As seen in the postoperative 3D CT reconstruction in Figure 4, a large gap remains on the anterior wall. Due to the poor quality of this reduction, this patient eventually had to be re-operated. The simulation led to a similar surgical failure. The compression clamp produced an adequate reduction of the posterior wall, however the large anterior gap is clearly visible in our model. While not quantified, this outcome is qualitatively completely coherent with the actual postoperative outcome. In addition, it appeared clearly during simulation that the ischium and femoral head were in contact, the femur hence obstructing the reduction. The use of our simulator may thus have prevented the surgeon to proceed with this gesture. The planning should have been changed to an anterior approach with femoral distraction, which was indeed done in a second surgery. Figure 5 shows the simulation of this later surgery, which yield results similar to the actual outcome.
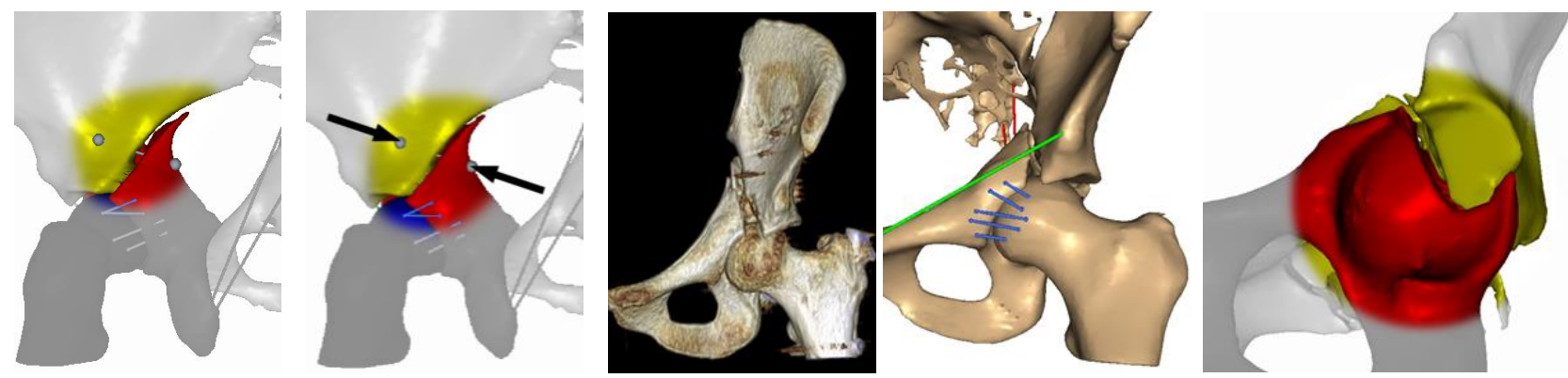

Figure 4. Clinical case \#2 (transverse fracture), first intervention from a Kocher-Langenbeck posterior approach. A single posterior clamp was used, however the femur was not distracted which eventually blocked the inferior fragment. An important gap can be seen on the anterior wall of the acetabulum, on both the postoperative 3D CT reconstruction and the simulation outcome. With this simulator available, the planning should have been changed to an anterior approach with femoral distraction, such as simulated Figure 5.
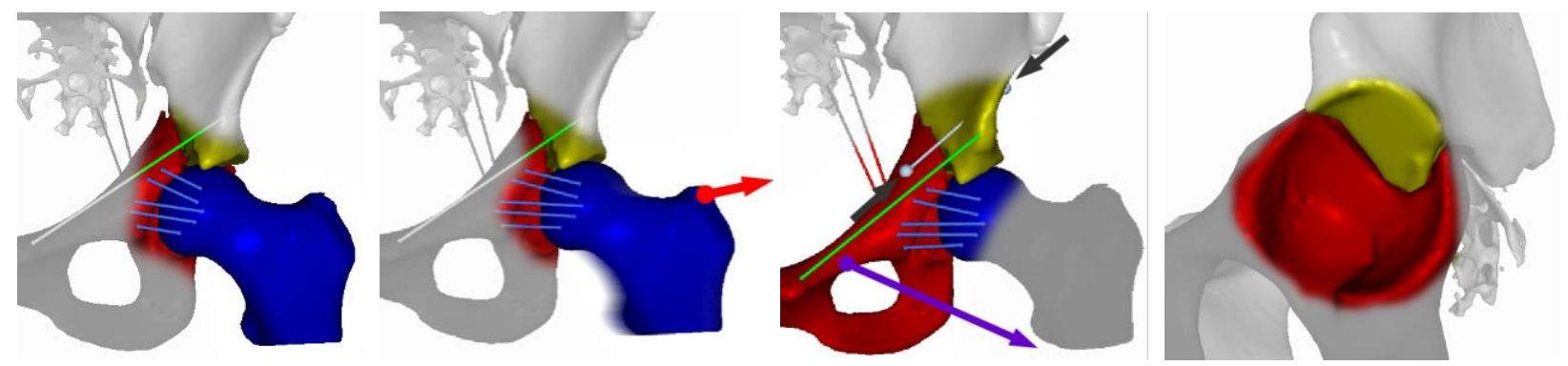

Figure 5. Clinical case \#2, second intervention from an anterior ilioinguinal approach. From left to right: combination of a femoral traction, an anterior clamp and pulling with a Picador pike. Although not fully anatomical, the reduction is adequate and qualitatively corresponds to the real outcome of this second surgery. 
Figure 6 presents a more complex case, with several gestures simulated: femoral distraction, direct impaction of the anterior column, two forceps. The results is here again coherent with the actual outcome. In a planning process, such result could be used by the surgeon to ensure the feasibility and relevancy of a given strategy.
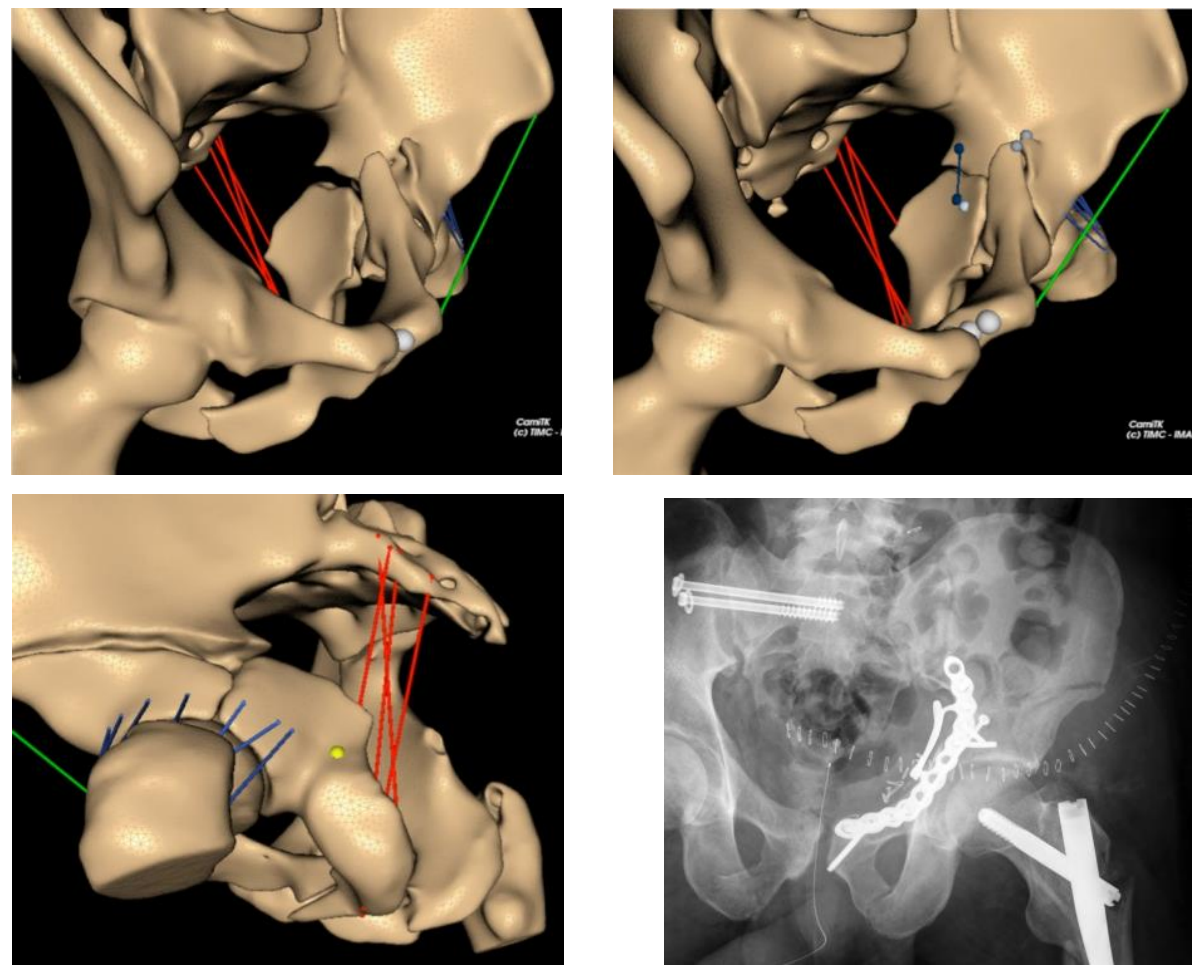

Figure 6. Clinical case \#6, a bi-columns fracture presented Figure 1. From left to right: before then after reduction (anterior and posterior views) from an anterior Stoppa-Cole surgical approach. This simulation showed that the reduction can be successfully obtained via this mini-invasive approach, including in the posterior part which is not accessible during the surgery. This result was confirmed by the actual surgical process and outcome.

For each case, simulation reproduced the actual surgical outcome with excellent fidelity, according to two different surgeons. Despite being preliminary, these results are promising and prove the soundness of our approach. A quantitative analysis is currently being run, to measure distances between landmarks on the simulated model and postoperative data.

\subsection{Clinical practicability}

Thanks to the specifically designed GUI, the patient anatomical model is built in a few minutes. The user just has to interactively pick the insertions for each spring ligament. Although they can be modified, default mechanical parameters were always used. Finally, reproducing the surgery is also a matter of minutes. Once the interactions are set (clamps, ...) numerical simulation is ran in real time on a standard laptop. Dynamically, the model behavior is adequate with reality, and has shown good repeatability.

The only long part remains the CT segmentation of bone fragments. ITK-Snap (www.itksnap.org) was used to perform thresholding, region growing then manual refinements. Models of adequate quality were obtained in 30 to 180 minutes, depending on the fracture complexity. These values are similar to the ones reported in [3,4]. Other groups have addressed the segmentation using promising semi-automatic methods [12,13], although the problem remains complex.

\subsection{Future works}

Works in progress are a better representation of the hip capsule, with non-straight ligaments that could wrap around osseous structures or with a continuous Finite-Element model. Some deep muscles may also be integrated, instead of a global damping. The graphical user interface will be improved to account for reduced visibility of surgical approaches. Finally, more cases will be included, especially with complex pattern, and the added clinical value will be assessed. 


\section{CONCLUSION}

Biomechanical simulation provides a novel approach for computer assisted acetabular fracture treatment. A multibody muskuloskeletal model of the hip was proposed, in a patient-specific context. This method is compatible with a clinical use, thanks to limited interactions and interactive computation times, the lone critical limitation being CT segmentation.

The first breakthrough is our paradigm, which differs from other authors: acetabular surgery itself is simulated, not its expected outcome. The second is the biomechanical simulation, which easily enables to reproduce actual actions of the surgeon and evaluate their outcomes. The proposed muskuloskeletal model is extremely simple in comparison with the literature. Yet, is was actually carefully designed to be simple but very specific to this given application. Although only qualitatively evaluated, first results fulfill our requirements: patient-specific, compatible with a clinical use, and good predicting capability. This biomechanical simulation tool to actually plan surgery will now have to be accessed.

\section{ACKNOWLEDGMENTS}

This work was partly supported by the french ANR within the Investissements d'Avenir program (labex CAMI) under reference ANR-11-LABX-0004.

\section{REFERENCES}

[1] Judet, R., Judet, J. and Letournel, E., "Fractures of the acetabulum: classification and surgical approaches for open reduction", The Journal of Bone \& Joint Surgery 46(8), 1615-1675 (1964).

[2] Boudissa, M., Ruatti, S., Kerschbaumer, G., Milaire, M., Merloz, P. and Tonetti, J., "Part 2: outcome of acetabular fractures and associated prognostic factors-a ten-year retrospective study of one hundred and fifty six operated cases with open reduction and internal fixation", Int Orthop. 40, 2151-2156 (2016)

[3] Cimerman, M. and Kristan, A., "Preoperative planning in pelvic and acetabular surgery: the value of advanced computerised planning modules", Injury 38(4), 442-449 (2007).

[4] Fornaro, J., Keel, M., Harders, M., Marincek, B., Szekely, G. and Frauenfelder, T., "An interactive surgical planning tool for acetabular fractures: initial results", J Orthop Surg Res 5(1), 50-55 (2010).

[5] Hu, Y., Li, H., Qiao, G., Liu, H., Ji, A. and Ye, F., "Computer-assisted virtual surgical procedure for acetabular fractures based on real ct data", Injury 42(10), 1121-1124 (2011).

[6] Lee, P. Y., Lai, J. Y., Yu, S. A., Huang, C. Y., Hu, Y. S. and Feng, C. L., "Computer-assisted fracture reduction and fixation simulation for pelvic fractures", Journal of Medical and Biological Engineering, 34, 368-376, 2014.

[7] Delp, S. L., Loan, J. P., Hoy, M. G., Zajac, F. E., Topp, E. L. and Rosen, J. M., "An interactive graphics-based model of the lower extremity to study orthopaedic surgical procedures", IEEE Transactions on Biomedical Engineering 37(8), 757-767 (1990).

[8] Blemker, S. S. and Delp, S. L., "Three-dimensional representation of complex muscle architectures and geometries", Annals of biomedical engineering 33(5), 661-673 (2005).

[9] Gilles, B. and Magnenat-Thalmann, N., "Musculoskeletal mri segmentation using multiresolution simplex meshes with medial representations", Medical image analysis 14(3), 291-302 (2010).

[10]Elkins, J., Stroud, N., Rudert, J., Tochigi, Y., Pedersen, D., Ellis, B., Callaghan, J., Weiss, J. and Brown, T., "The capsule's contribution to total hip construct stability a finite element analysis", J. Orthop. Res. 29, 16421648 (2011).

[11]Lloyd, J.E., Stavness, I. and Fels, S., "Artisynth: a fast interactive biomechanical modeling toolkit combining multibody and finite element simulation", In: Soft tissue biomechanical modeling for computer assisted surgery, 355-394, Springer (2012).

[12] Fornaro, J., Szekely, G. and Harders, M., "Semi-automatic segmentation of fractured pelvic bones for surgical planning", International Symposium on Biomedical Simulation, 82-89, Springer Berlin Heidelberg (2010)

[13] Wu, J., Davuluri, P., Ward, K.R., Cockrell, C., Hobson, R. and Najarian, K., "Fracture detection in traumatic pelvic ct images", Journal of Biomedical Imaging 1 (2012). 